\title{
Hereditary diffuse gastric cancer: strategies to reduce tumoral risk
}

\author{
Maite Herráiz ${ }^{1}$, Víctor Valenti², Jesús Sola ${ }^{3}$, Pilar Pérez-Rojo ${ }^{1}$, Fernando Rotellar ${ }^{2}$ \\ and Javier A.-Cienfuegos ${ }^{2}$ \\ ${ }^{1}$ Prevention Unit and High-Risk Gastrointestinal Cancer Clinic. Department of Gastroenterology. \\ ${ }^{2}$ Department of Surgery. ${ }^{3}$ Department of Pathology. University Clinic of Navarra. Pamplona, Navarra. Spain
}

\begin{abstract}
The main goal at a High-Risk Gastrointestinal Cancer Clinic is to identify individuals at increased risk of developing tumors for diagnosis them in presymptomatic stages, when they are potentially curable. We report an asymptomatic patient belonging to a family with hereditary diffuse gastric cancer syndrome with a novel pathogenic mutation in the E-cadherin gene. In the absence of any proven diagnostic tool in surveillance tumor of this syndrome, the recommendation accepted today for an asymptomatic individual with known mutation is to perform prophylactic surgery. This patient underwent total laparoscopic gastrectomy. A microscopic focus of tumor was detected in the surgical specimen. Strategies to reduce the tumor risk in the hereditary diffuse gastric cancer syndrome are limited, but it is necessary to recognize them in order to treat these patients accordingly to the available evidence.
\end{abstract}

Key words: Hereditary cancer. Gastric cancer. Hereditary gastric cancer. Prophylactic gastrectomy. E-cadherin. CDH1.

Herráiz M, Valenti V, Sola J, Pérez-Rojo $P$, Rotellar $F$ and A. Cienfuegos J. Hereditary diffuse gastric cancer: strategies to reduce tumoral risk. Rev Esp Enferm Dig 2012; 104: 326-329.

\section{INTRODUCTION}

Hereditary diffuse gastric cancer (HDGC) is defined as a syndrome of inherited predisposition to cancer with an autosomal dominant inheritance pattern. HDGC is caused by germline mutation of the $\mathrm{CDH} 1$ gene that encodes the cell adhesion protein E-cadherin (1). CDH1 gene mutations have

Received: 11-01-2012

Accepted: 14-02-2012

Correspondence: Maite Herráiz. Prevention Unit and High-Risk. Gastrointestinal Cancer Clinic. Department of Gastroenterology. Clínica Universidad de Navarra. Avda. Pío XII, 36. 31009 Pamplona. Navarra, Spain e-mail: mherraizb@unav.es a high penetrance so that mutation carriers have a more than $80 \%$ lifetime risk of developing gastric cancer and an elevated risk of lobular breast cancer (2). This very high risk of gastric cancer demands prevention strategies, which remain the main problem in the management of families with HDGC (3). Thus far, no study has demonstrated the effectiveness of different imaging techniques in surveillance these patients including: upper endoscopy with multiple biopsies, high-definition endoscopy, chromoendoscopy, endoscopic ultrasonography, computed tomography and abdominal positron emission tomography (4). Clinical management of HDGC involves predictive genetic testing. Moreover, it is recommended that mutation carriers undergo prophylactic total gastrectomy due to the almost universal presence of microscopic foci of tumor in these individuals (3).

We report the case of a woman who came to our clinic for several first-degree relatives with diffuse gastric cancer.

\section{CASE REPORT}

A 51-year old woman was referred to our clinic for evaluation of gastric cancer risk. She had no relevant past medical history and she was asymptomatic. Her family history (Fig. 1) was remarkable for gastric cancer: the oldest brother was diagnosed with gastric adenocarcinoma with signet ring cells at age 57 and died a year later. The next brother was diagnosed with disseminated gastric adenocarcinoma diffuse type at age 56, and died within months. A 54-year-old sister had just been diagnosed with diffuse adenocarcinoma of the stomach with signet-ring cells, having bilateral ovarian involvement at diagnosis and peritoneal carcinomatosis. This sister had undergone an upper endoscopy six months earlier for an abdominal discomfort without any relevant findings. She had another younger sister of 49 years, healthy, and two healthy sons. Her father died at age 91 with a prostate tumor diagnosed 17 years before and her mother was still alive (89 years) without tumoral disease. 


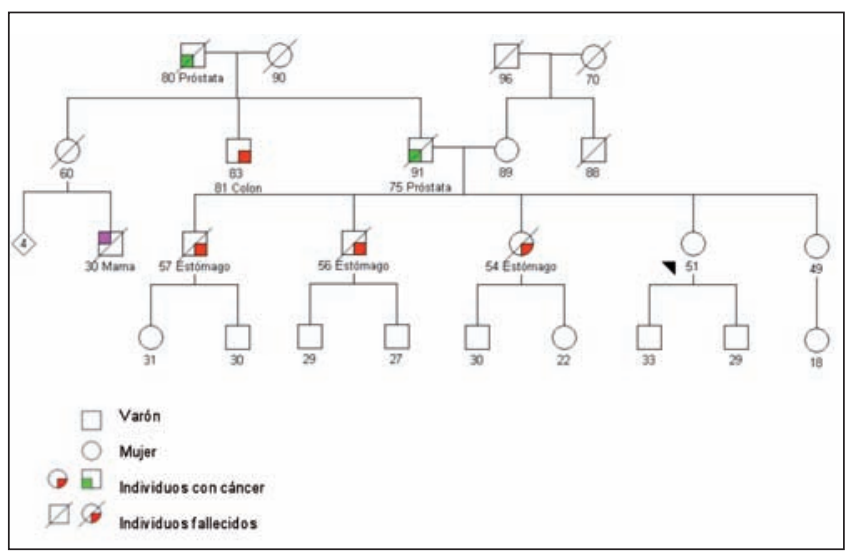

Fig. 1. Patient's pedigree at the time of the consultation. The proband is marked with an arrow.

In the presence of three first-degree relatives diagnosed with diffuse-type gastric adenocarcinoma (confirmed in pathology reports provided by the patient) in a relatively young age she was advised to rule out a HDGC. Sequencing of CDH1 gene was performed in her sister newly diagnosed of stomach cancer. A novel c.336delC mutation in exon 3 was found. This mutation generates a premature stop codon at position 117 giving rise to a truncated protein, thus considered as the cause of gastric cancer risk. The presence of a c.336delC germline mutation was confirmed in the asymptomatic consultant patient. The youngest sister was negative for the mutation.

The patient underwent extensive evaluation that included complete history and physical examination, standard laboratory studies, upper gastrointestinal endoscopy with biopsy using a high-definition endoscope with NBI (Olympus GIFH180) and endoscopic ultrasonography to rule out the presence of macroscopic lesions. Both techniques were normal. After appropriate genetic counseling, a prophylactic total gastrectomy was performed laparoscopically without lymphadenectomy. The margins of section included esophageal and duodenal tissue needed to confirm the complete removal of the gastric mucosa. The procedure lasted 190 minutes and did not require blood transfusion. On the fifth postoperative day radiological control was performed with oral contrast to exclude the presence of leaks and the patient was discharged seven days after surgery. Upon receipt of the specimen in surgical pathology, stomach was opened along the greater curvature (Fig. 2), digitally photographed, pinned to a cork board, and fixed in $10 \%$ formalin for 48 hours. After fixation, serial cuts of the specimen (each section $2 \mathrm{~cm} \times 0.3 \mathrm{~cm}$ thick), were taken obtaining a total of 140 blocks with three pieces included in each block. The esophageal, fundic and body, the transition zone body-antrum, antrum and duodenal type mucosa were identified. In a section of the posterior wall of the fundus, close to the cardia, a small focus of adenocarcinoma was identified. This focus of adenocarcinoma exhibited characteristic signet ring cell morphology, showing the cytoplasm of epithelial cells with abundant mucin with the nucleus compressed to the periphery. Neoplastic cells were confined to

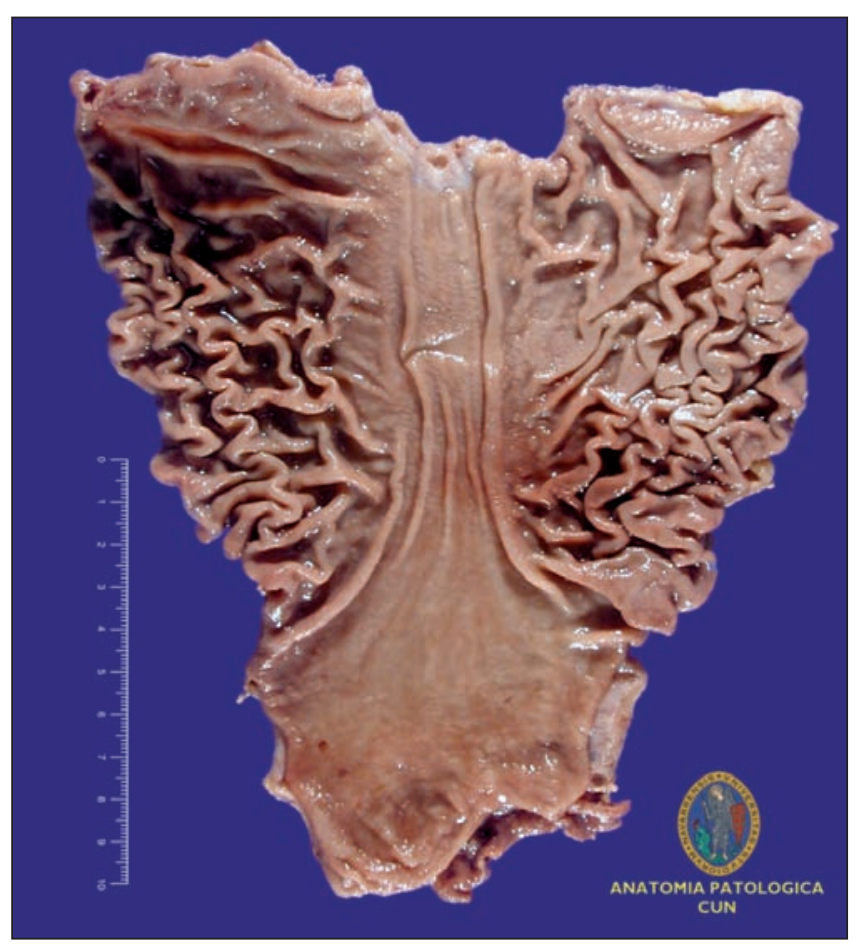

Fig. 2. Macroscopic image of the total gastrectomy specimen without visible changes in the gastric mucosa in the patient with a mutation in $\mathrm{CDH} 1$.

the superficial portion of the lamina propria and the size was $0.250 \mathrm{~mm}$. No extension beyond the muscularis mucosa was identified (stage pT1A pNx pMX) (Fig. 3).

\section{DISCUSSION}

As happens with colon cancer, there have also been described specific syndromes of inherited gastric cancer with a very high risk for developing this type of tumor. Due to

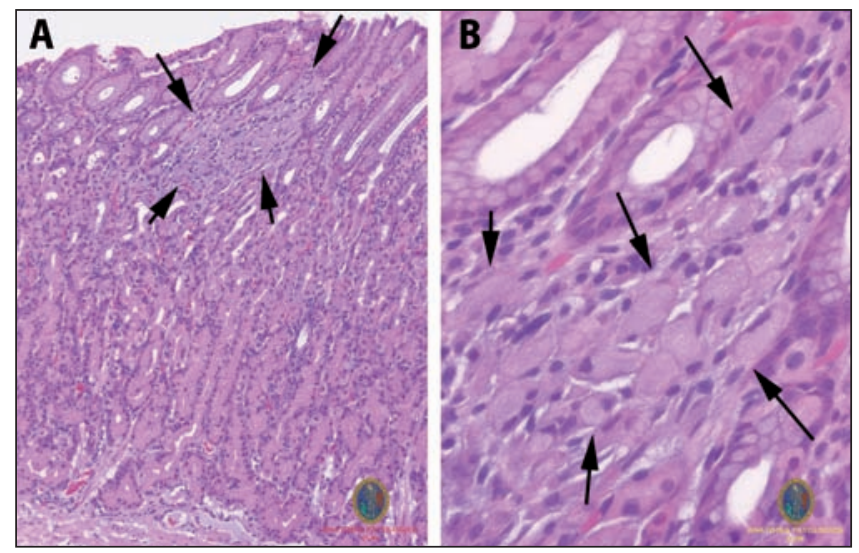

Fig. 3. A. $0.250 \mathrm{~mm}$ focus of intramucosal cell diffuse signet-ring carcinoma infiltrating between the crypts (indicated by arrows) and normal gastric epithelium in the superficial lamina propria $(x 40)$. B. Focus of occult carcinoma of signet ring cells (400x). Hematoxylin and eosin. 
the growing number of high-risk gastrointestinal tumor clinics, it become necessary to recognize these syndromes in order to reliably identify individuals who potentially may have an increased risk of developing a tumor and manage them accordingly to the current guidelines.

Gastric cancer is the fourth most common cause of cancer globally. The vast majority of gastric cancers are sporadic, although it is well established that approximately 1$3 \%$ of tumors arise as a result of inherited gastric cancer predisposition syndromes (5). Inherited gastric cancers are most often of the diffuse type or linitis plastica and are generally referred to as hereditary diffuse gastric cancer. In order for a family to qualify for a diagnosis of HDCG the following criteria have been used $(6,7)$ :

- Two or more documented cases of diffuse gastric cancer in first or second degree relatives, with at least one diagnoses before the age of 50, or

- Three or more cases of documented diffuse gastric cancer in first/second degree relatives, independent of age of onset.

Linkage studies showed a decade ago, that this syndrome is caused by germline mutations in the gene suppressor E-cadherin (CDH1) (1). It is estimated that approximately $25 \%$ of families that meet the criteria set out above will have an inactivating germline CDH1 (6). Published data from these families suggest that the penetrance of $\mathrm{CDH} 1$ mutations is high (>80\%) (5), so that carriers of these mutations have a very high risk of developing gastric cancer. Germline mutations in E-cadherin also predispose to the development of other tumors such as lobular breast cancer and possibly colon cancer.

Clinical management in HDGC is troublesome. A consensus conference of experts lately updated consensus clinical guidelines for the management of these patients (5). This paper place particular emphasis on the need for patients with suspected CGHD are monitored by multidisciplinary teams that include gastrointestinal endoscopists, surgeons and pathologists with expertise in this syndrome.

Genetic counseling is an essential component of the evaluation and management of HDGC. The genetic evaluation should include a careful three-generation family pedigree, histopathological confirmation of the diagnosis of diffuse gastric cancer, a detailed discussion of the lifetime risk of developing stomach cancer and the possibilities of surveillance available today. Genetic testing should begin in a familiar with cancer. In our case, the study was indicated in the only sister diagnosed with gastric cancer who was alive at the time of the consultation. $\mathrm{CDH} 1$ sequencing found a previously unidentified mutation that was considered pathological for producing a stop codon resulting in a truncated protein. The discovery of a pathological mutation in an affected family member extends the analysis to others first-degree relatives. The two sisters at risk were performed direct mutational analysis, being positive in one. A negative result, not finding the mutation, is considered a true negative when there is a known mutation in the family. Those individuals have no more risk than the general population and need not undergo an intensive tumor surveillance and/or prophylactic surgery.

Once an individual has been identified as a healthy carrier of a disease-causing mutation, the advice is to consider performing prophylactic total gastrectomy regardless of the endoscopy findings (3). The time of gastrectomy may vary depending on patient preference, age, physical status, etc. In our case the prophylactic gastrectomy was recommended without delay considering the patient's age and age at diagnosis of tumors in her siblings. Prior to surgery is recommended to perform an upper endoscopy to detect gross lesions. Gastroscopy was performed looking carefully in the subcardial area by the tendency to accumulate in this area many microscopic foci of tumor (8), but found no abnormalities. Multiple random biopsies were taken that were negative for tumor. Endoscopic ultrasonography was performed seeking pathological thickening of the wall (linitis plastica) without relevant findings.

The requisite operation is a total gastrectomy with Rouxen-Y reconstruction. The proximal resection line must be across the distal esophagus to ensure that no gastric cardia mucosa is left behind. In the setting of prophylactic surgery does not seem necessary radical lymph node dissection due to minimal risk of lymph node metastases of adenocarcinoma confined to the mucosa without involvement of the submucosa (9). There are no available studies comparing the surgical approach, open gastrectomy to laparoscopic, although the recommendation of the consensus guide (5) is that the laparoscopic approach should be done in experienced centers without increased risk for the patient. In this case total gastrectomy was performed by laparoscopy. To date there are only six cases of laparoscopic prophylactic gastrectomy published, one of them laparoscopically assisted $(10,11)$.

Currently, 96 cases of prophylactic total gastrectomy in the setting of HDGC have been published. Seventy-three cases are, as the case presented, real prophylactic gastrectomies, that is, performed in individuals carrying a mutation in $\mathrm{CDH} 1$ gene with negative studies before surgery. Of the total, 42 surgical specimens were studied with a specific protocol, identifying early carcinoma signet-ring cells in 39 cases $(93 \%)$. In the remaining 31 specimens studied without a specific protocol, were identified a total of 25 early gastric cancers $(81 \%)(5)$. In our case the analysis was following the protocol recommended and identified a microscopic focus of tumor 250 microns in diameter located in the posterior wall of fundus, near the cardia. The treatment is considered curative at this stage.

To our knowledge, this patient represents the first case of HDGC detected by prophylactic gastrectomy in Spain. Despite its low frequency highlights the complexity in the care of these patients and their families and stresses the need for multidisciplinary teams with experience in this disease. 


\section{REFERENCES}

1. Guilford PJ, Hopkins JB, Grady WM, Markowitz SD, Willis J, Lynch H, et al. E-cadherin germline mutations define an inherited cancer syndrome dominated by diffuse gastric cancer. Hum Mutat 1999;14(3):249-55.

2. Pharoah PD, Guilford P, Caldas C, International Gastric Cancer Linkage Consortium. Incidence of gastric cancer and breast cancer in $\mathrm{CDH} 1$

(E-cadherin) mutation carriers from hereditary diffuse gastric cancer families. Gastroenterology 2001;121(6):1348-53.

3. Blair V, Martin I, Shaw D, Winship I, Kerr D, Arnold J, et al. Hereditary diffuse gastric cancer: diagnosis and management. Clin Gastroenterol Hepatol 2006;4(3):262-75.

4. Norton JA, Ham CM, Van Dam J, Jeffrey RB, Longacre TA, Huntsman $\mathrm{DG}$, et al. CDH1 truncating mutations in the E-cadherin gene: an indication for total gastrectomy to treat hereditary diffuse gastric cancer. Ann Surg 2007;245(6):873-9.

5. Fitzgerald RC, Hardwick R, Huntsman D, Carneiro F, Guilford P, Blair $\mathrm{V}$, et al. Hereditary diffuse gastric cancer: updated consensus guidelines for clinical management and directions for future research. J Med Genet 2010;47(7):436-44
6. Caldas C, Carneiro F, Lynch HT, Yokota J, Wiesner GL, Powell SM, et al. Familial gastric cancer: overview and guidelines for management. J Med Genet 1999;36(12):873-80.

7. Oliveira C, Bordin MC, Grehan N, Huntsman D, Suriano G, Machado $\mathrm{JC}$, et al. Screening E-cadherin in gastric cancer families reveals germline mutations only in hereditary diffuse gastric cancer kindred. Hum Mutat 2002;19(5):510-7.

8. Rogers WM, Dobo E, Norton JA, Van Dam J, Jeffrey RB, Huntsman DG, et al. Risk-reducing total gastrectomy for germline mutations in E-cadherin (CDH1): pathologic findings with clinical implications. Am J Surg Pathol 2008;32(6):799-809.

9. Sano T, Kobori O, Muto T. Lymph node metastasis from early gastric cancer: endoscopic resection of tumour. Br J Surg 1992;79(3):241-4.

10. Francis WP, Rodrigues DM, Perez NE, Lonardo F, Weaver D, Webber JD. Prophylactic laparoscopic-assisted total gastrectomy for hereditary diffuse gastric cancer. JSLS 2007;11(1):142-7.

11. Mayrbaeurl B, Keller G, Schauer W, Burgstaller S, Czompo M, Hoebling W, et al. Germline mutation of the E-cadherin gene in three sibling cases with advanced gastric cancer: clinical consequences for the other family members. Eur J Gastroenterol Hepatol 2010;22(3):306-10. 